\title{
Piezoelectric polymer thin films with architected cuts - CORRIGENDUM
}

Lichen Fang, Jing Li, Zeyu Zhu, Santiago Orrego, and Sung Hoon Kang

doi: 10.1557/jmr.2018.6, Published by Materials Research Society with Cambridge University Press, 14 February 2018.

In the final paragraph on page 331 in Fang et al., ${ }^{1}$ sentence reading "Recently, the fractal cut has been developed to introduce even higher stretchability compared to other cut patterns," lists the incorrect reference number. The reference cited should be 18 instead of 38 .

The authors apologize for this error.

\section{REFERENCE}

1. L. Fang, J. Li, Z. Zhu, S. Orrego, and S.H. Kang: Piezoelectric polymer thin films with architected cuts. J. Mater. Res. 33, 330-342 (2018). 